\title{
Overexpression of POSTN in Tumor Stroma Is a Poor Prognostic Indicator of Colorectal Cancer
}

Hyeon Jeong $\mathrm{Oh}^{1,2} \cdot$ Jeong Mo $\mathrm{Bae}^{2,3}$ Xian-Yu Wen ${ }^{2} \cdot \mathrm{Nam}-\mathrm{Yun} \mathrm{Cho}^{2}$ Jung Ho Kim ${ }^{1,2}$. Gyeong Hoon Kang ${ }^{1,2}$

'Department of Pathology, Seoul National University College of Medicine, Seoul; 'Laboratory of Epigenetics, Cancer Research Institute, Seoul National University College of Medicine, Seoul; ${ }^{3}$ Department of Pathology, SMG-SNU Boramae Medical Center, Seoul, Korea

Received: October 11, 2016

Revised: November 30, 2016

Accepted: January 19, 2017

\section{Corresponding Author}

Gyeong Hoon Kang, MD

Department of Pathology, Seoul National University

College of Medicine, 103 Daehak-ro, Jongno-gu,

Seoul 03080, Korea

Tel: +82-2-740-8263

Fax: +82-2-765-5600

E-mail: ghkang@snu.ac.kr
Background: Tumor microenvironment has recently drawn attention in that it is related with tumor prognosis. Cancer-associated fibroblast also plays a critical role in cancer invasiveness and progression in colorectal cancers. Periostin (POSTN), originally identified to be expressed in osteoblasts and osteoblast-derived cells, is expressed in cancer-associated fibroblasts in several tissue types of cancer. Recent studies suggest an association between stromal overexpression of POSTN and poor prognosis of cancer patients. Methods: We analyzed colorectal cancer cases for their expression status of POSTN in tumor stroma using immunohistochemistry and correlated the expression status with clinicopathological and molecular features. Results: High level of POSTN expression in tumor stroma was closely associated with tumor location in proximal colon, infiltrative growth pattern, undifferentiated histology, tumor budding, luminal necrosis, and higher TNM stage. High expression status of POSTN in tumor stroma was found to be an independent prognostic parameter implicating poor 5-year cancer-specific survival and 5-year progression-free survival. Conclusions: Our findings suggest that POSTN overexpression in tumor stroma of colorectal cancers could be a possible candidate marker for predicting poor prognosis in patients with colorectal cancers.

Key Words: Colorectal neoplasms; POSTN; Immunohistochemistry; Stromal overexpression; Clinicopathological characters; Prognosis
Colorectal cancer (CRC) is the third most common cancer in the world, with nearly 1.4 million new cases diagnosed in 2012, and South Korea is one of the countries with the highest incidence of CRC. ${ }^{1}$ Although TNM staging of American Joint Committee on Cancer (AJCC) is the most powerful and reliable tool for prediction of prognosis and therapeutic decision making in CRC patients, the clinical outcome of CRC patients may vary even within the same cancer stage. Despite curative surgery and adjuvant chemotherapy and/or radiation therapy, approximately $17 \%$ of stage II CRCs and $33 \%$ of stage III CRCs will have a disease recurrence following primary therapy. ${ }^{2}$ Identification of CRCs with a high risk of recurrence might give a chance of benefit from additional therapy. Many studies are trying to find factors that could more precisely stratify patients into different risk categories.

Recent studies find that tumor microenvironment and tumorstroma interaction are critical in tumor behavior. In tumor microenvironment, cancer-associated fibroblasts have an impor- tant role in tumor-stroma interaction and could affect prognosis. ${ }^{3-7}$ However, there are no specific markers of cancer-associated fibroblasts that have been proven to be related with tumor prognosis. Periostin (POSTN) is a secreted extracellular matrix protein which is originally identified to be expressed in osteoblasts and osteoblast-derived cells. POSTN is normally expressed in not only collagen-rich fibrous connective tissues, including periosteum and periodontal ligament, but also fibroblasts of normal tissue, including stomach and colon. ${ }^{8}$ POSTN is also expressed in cancer-associated fibroblasts of breast, colon, lung, pancreas, and stomach cancer., ${ }^{9,10}$ POSTN binds integrins, including $\alpha v \beta 3, \alpha v \beta 5$, and $\alpha 6 \beta 4$, which leads to the activation of the Akt/protein kinase B and focal adhesion kinase signalling pathways. ${ }^{11-13}$ POSTN-activated signalling pathways promote angiogenesis, cellular survival, and resistance to hypoxia-induced cell death. ${ }^{8,14}$ POSTN is also involved in epithelial-mesenchymal transition of tumor. ${ }^{8}$ Recent studies show that POSTN is associated with tumor invasiveness and poor prognosis in 
several malignancies. ${ }^{15-17}$

In the present study, we analyzed 1,125 CRC cases for their expression status of POSTN using immunohistochemistry and correlated POSTN expression with molecular features of CRCs, including $\mathrm{CpG}$ island methylator phenotype (CIMP), microsatellite instability (MSI), and clinicopathological features of CRC, including survival of patients. We tried to identify whether POSTN expression in cancer-associated fibroblast can be a prognostic marker in CRC.

\section{MATERIALS AND METHODS}

\section{Tissue samples}

A consecutive series of CRC cases were retrieved from the surgical files of the Department of Pathology, Seoul National University Hospital, Seoul, Korea. Among the patients who underwent surgical resection for primary CRC from 2004 to 2007, we excluded those with neo-adjuvant treatment, non-invasive cancers, familial adenomatous polyposis, multiple or recurrent tumors, and a history of other malignancy within 5 years. Demographic data and clinicopathological information were retrieved from electronic medical records. Two pathologists (J.M.B. and G.H.K.) reviewed hematoxylin and eosin-stained tissue slides for the degree of histologic differentiation which was categorized as well-moderate versus poor ( $>50 \%$ vs $\leq 50 \%$, respectively). Staging was classified according to the sixth edition guidelines of the AJCC. Hematoxylin and eosin-stained slides were also reviewed for evaluation of tumor budding, which is defined to be present when five or more buddings are present at $\times 200$ magnification. Status of tumor infiltrating lymphocytes was divided into high and low ( $\geq 8$ at $\times 400$ magnification and $<8$ at $\times 400$ magnification, respectively). The study was approved by Institutional Ethics Committee of Seoul National University Hospital which waived the requirement to obtain informed consent (approval No. 1502-029-647).

\section{Evaluation of POSTN expression}

After reviewing the hematoxylin and eosin tissue slides, representative areas of the tumor invasive front were selected and marked. Two-millimeter-core tissues were harvested from individual paraffin-embedded colon cancer tissues and were arranged in a new recipient paraffin block using a trephine apparatus (Superbiochip Laboratories, Seoul, Korea). Immunohistochemical analysis was performed with commercially available antibody against POSTN (1:200, HPA012306, Sigma, St. Louis, MO, USA). Two pathologists (H.J.O. and J.M.B.) independently evaluated POSTN immunohistochemistry. POSTN expression in tumor stroma showed homogeneous staining intensity and the level of POSTN expression was graded as 0 (negative), 1 (weak), 2 (moderate), and 3 (strong) (Fig. 1). Then, grades $0-2$ and 3 were categorized as POSTN-low and POSTN-high, respectively.

\section{$B R A F$ and $K R A S$ mutation and microsatellite instability analysis}

Through microscopic examination, representative tumor areas in each case were marked and microdissected. The dissected tissues were subject to incubation at $55^{\circ} \mathrm{C}$ with lysis buffer and proteinase $\mathrm{K}$ for 2 days. Allele specific polymerase chain reaction for $B R A F$ codon 600 and direct sequencing of KRAS codons 12 and 13 were performed. The MSI status of each tumor tissue versus normal tissue was determined by five National Cancer Institute markers including BAT25, BAT26, D2S123, D5S346, and D17S250. High MSI status was defined as when tumor DNA had altered alleles compared to normal DNA in two or more markers. Low MSI status was defined as when tumor DNA had altered allele compared to normal DNA in one marker. Microsatellite stable was defined as when no altered allele was present in tumor DNA.

\section{CIMP analysis}

CIMP status was examined by MethyLight assay. Bisulfitemodified DNA was subject to MethyLight assay which was performed as previously described. ${ }^{18}$ Methylation statuses of eight CIMP-specific CpG islands (CACNA1G, CDKN2A, CRABP1, IGF2, MLH1, NEUROG1, RUNX3, and SOCS1) were quantified. CIMP-high was defined as five or more markers methylated, and CIMP-low was defined as four or less markers methylated out of eight. CIMP-0 was defined as no methylated marker .

\section{Statistical analysis}

SAS software (ver. 9.4 for Microsoft Windows, SAS Institute Inc., Cary, NC, USA) was used for the statistical analysis in our cohort. To compare clinicopathologic characteristics to stromal POSTN expression, we performed Pearson's chi-square test. The age of CRC group according to stromal POSTN expression was compared using Wilcoxon's rank-sum test. For the survival analysis, 5-year cancer-specific survival (CSS) and 5-year progression-free survival (PFS) were calculated using the log-rank test with a Kaplan-Meier curve. Hazard ratios (HRs) were calculated using the Cox proportional hazard model. The assumption of the proportional hazards was verified by plotting the $\log \{-\log [\mathrm{S}(\mathrm{t})]\}$ 

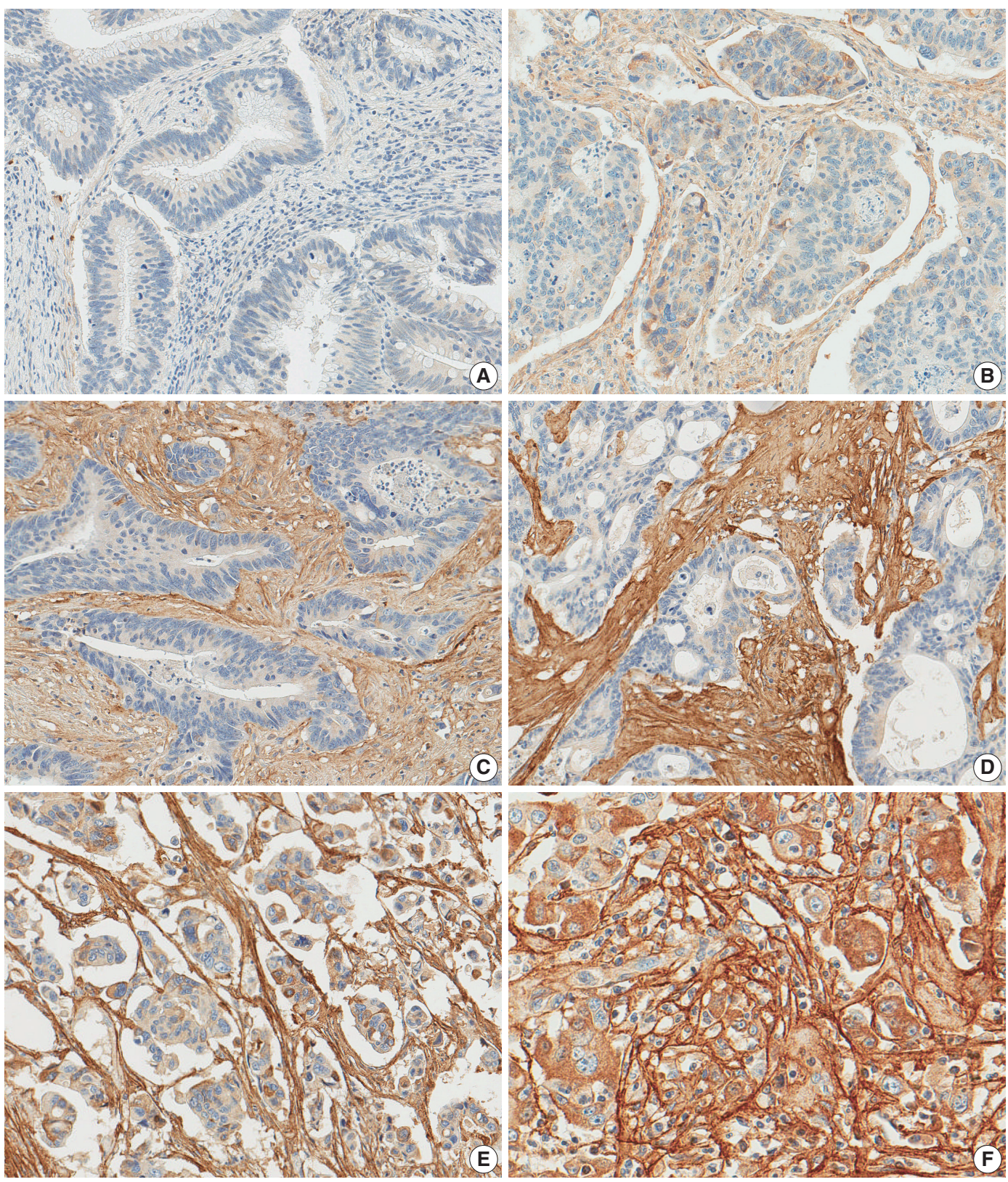

Fig. 1. Periostin expression in colorectal cancer. Grade $0(A)$, grade $1(B)$, grade $2(C)$, and grade $3(D)$ in moderately differentiated adenocarcinoma, grade 3 in poorly differentiated adenocarcinoma (E), grade 3 in high tumor budding area (F).

against the time of the study. In the modeling process, all variables that were associated with PFS with a $\mathrm{p}<.10$ were entered into an initial model; these variables were subsequently reduced by backward elimination. All statistical tests were two-sided, and statistical significance was defined as $\mathrm{p}<.05$.

\section{RESULTS}

\section{Patient characteristics}

In a total of 1,135 CRC patients, the median age at diagnosis was 62 years (range, 20 to 90 years). The male to female ratio was 1.49:1 (673 males and 452 females). Tumor location was proximal colon (proximal to splenic flexure) in 277 patients (24.6\%), distal colon in 438 patients (38.9\%), and rectum in 410 patients (36.4\%). KRAS mutation and BRAF mutation were observed in $313(27.8 \%)$ and 48 patients (4.3\%), respectively. In microsatellite analysis, microsatellite stable, MSI-low, and MSI-high were observed in $964(85.7 \%), 73(6.5 \%)$, and $88(7.8 \%)$ patients, respectively. In CIMP analysis, CIMP-0, CIMP-low, and CIMP-high were observed in 510 (45.3\%), 553 (49.2\%), and 
$62(5.5 \%)$ patients, respectively. Median follow-up duration was 69.8 months (range, 0.3 to 150.2 months). Seven hundred seventy-nine patients received 5-fluorouracil (5-FU)-based adjuvant chemotherapy.

\section{Clinicopathological features of CRCs according to stromal POSTN expression}

Of 1,125 CRC cases, 33 (2.9\%), 296 (26.3\%), 492 (43.7\%), and $304(27.0 \%)$ patients showed stromal POSTN expression from grade 0 to 3, respectively. POSTN-low CRCs were 821 (73.0\%) and POSTN-high CRCs were 304 (27.0\%) (Table 1). CRCs with stromal POSTN-high expression were associated with proximal location (35.2\% in POSTN-high group vs $20.7 \%$ in POSTN-low group, $\mathrm{p}<.001$ ), infiltrative growth pattern (44.7\% vs $30.7 \%, \mathrm{p}<.001$ ), advanced T, N, M category ( $\mathrm{p}<$ $.001)$, frequent tumor budding $(80.9 \%$ vs $68.2 \%, \mathrm{p}<.001)$ and luminal necrosis $(94.4 \%$ vs $89.6 \%, \mathrm{p}=.014)$ compared with CRCs with stromal POSTN-low expression. In molecular aspect,

Table 1. Clinicopathologic characteristics of colorectal cancers according to the stromal POSTN expression

\begin{tabular}{|c|c|c|c|c|}
\hline Variable & & $\begin{array}{c}\text { POSTN-low } \\
(n=821,73.0 \%)\end{array}$ & $\begin{array}{c}\text { POSTN-high } \\
(n=304,27.0 \%)\end{array}$ & $p$-value \\
\hline Age (yr) & & $62(20-87)$ & $62(29-90)$ & .932 \\
\hline \multirow[t]{2}{*}{ Sex } & Male & $500(60.9)$ & $173(56.9)$ & .225 \\
\hline & Female & $321(39.1)$ & $131(43.1)$ & \\
\hline \multirow[t]{3}{*}{ Location } & Proximal & $170(20.7)$ & $107(35.2)$ & $<.001$ \\
\hline & Distal & $336(40.9)$ & $102(33.5)$ & \\
\hline & Rectum & $315(38.4)$ & 95 (31.3) & \\
\hline \multirow[t]{2}{*}{ Growth pattern } & Fungating & $569(69.3)$ & $168(55.3)$ & $<.001$ \\
\hline & Infiltrative & $252(30.7)$ & $136(44.7)$ & \\
\hline \multirow[t]{2}{*}{ T category } & $\mathrm{T} 1,2$ & $192(23.4)$ & $18(5.9)$ & $<.001$ \\
\hline & $\mathrm{T} 3,4$ & $629(76.6)$ & $286(94.1)$ & \\
\hline \multirow[t]{2}{*}{$\mathrm{N}$ category } & NO & $454(55.3)$ & $117(38.5)$ & $<.001$ \\
\hline & $\mathrm{N} 1,2$ & $367(44.7)$ & $187(61.5)$ & \\
\hline \multirow[t]{2}{*}{ M category } & 0 & $712(86.7)$ & $222(73.0)$ & $<.001$ \\
\hline & 1 & $109(13.3)$ & $82(27.0)$ & \\
\hline \multirow[t]{2}{*}{ Stage } & ।, II & $432(52.6)$ & $104(34.2)$ & $<.001$ \\
\hline & III, IV & $109(13.3)$ & $200(65.8)$ & \\
\hline \multirow[t]{2}{*}{ Differentiation } & Differentiated & $802(97.7)$ & $284(93.4)$ & .001 \\
\hline & Undifferentiated & $19(2.3)$ & $20(6.6)$ & \\
\hline \multirow[t]{2}{*}{ Tumor budding } & Absent & $261(31.8)$ & 58 (19.1) & $<.001$ \\
\hline & Present & $560(68.2)$ & $246(80.9)$ & \\
\hline \multirow[t]{2}{*}{ Dirty necrosis } & Absent & 85 (10.4) & $17(5.6)$ & .014 \\
\hline & Present & $736(89.6)$ & $287(94.4)$ & \\
\hline \multirow[t]{2}{*}{ Crohn-like reaction } & Absent & $703(85.6)$ & 251 (82.6) & .204 \\
\hline & Present & $118(14.4)$ & $53(17.4)$ & \\
\hline \multirow[t]{2}{*}{ Tumor-infiltrating lymphocytes } & High ( $\geq 8 / \mathrm{HPF})$ & $599(73.0)$ & $233(76.6)$ & .211 \\
\hline & Low (<8/HPF) & $222(27.0)$ & $71(23.4)$ & \\
\hline \multirow[t]{2}{*}{ Serration } & Absent & $793(96.6)$ & $288(94.7)$ & .155 \\
\hline & Present & $28(3.4)$ & $16(5.3)$ & \\
\hline \multirow[t]{2}{*}{ Mucin production } & Absent & $727(88.5)$ & $265(87.2)$ & .525 \\
\hline & Present & $94(11.5)$ & $39(12.8)$ & \\
\hline \multirow[t]{3}{*}{ CIMP } & CIMP-0 & $387(47.1)$ & $123(40.5)$ & .030 \\
\hline & CIMP-low & $396(48.2)$ & $157(51.6)$ & \\
\hline & CIMP-high & $38(4.6)$ & $24(7.9)$ & \\
\hline \multirow[t]{3}{*}{$\mathrm{MSI}$} & MSS & $702(85.5)$ & $262(86.2)$ & .959 \\
\hline & MSI-low & $54(6.6)$ & $19(6.2)$ & \\
\hline & MSI-high & $65(7.9)$ & $23(7.6)$ & \\
\hline \multirow[t]{2}{*}{ KRAS mutation } & Wild type & $602(73.3)$ & $210(69.1)$ & .158 \\
\hline & Mutant & $219(26.7)$ & $94(30.9)$ & \\
\hline \multirow[t]{2}{*}{ BRAF mutation $(n=1,124)$} & Wild type & $791(96.5)$ & $285(93.7)$ & .046 \\
\hline & Mutant & $29(3.5)$ & $19(6.3)$ & \\
\hline
\end{tabular}

Values are presented as median (range) or number (\%).

POSTN, periostin; HPF, high-power field; CIMP, CpG island methylator phenotype; MSI, microsatellite instability. 
CRCs with stromal POSTN-high expression showed higher frequency of CIMP-high $(7.9 \%$ vs $4.6 \%, \mathrm{p}=.030)$ and $B R A F$ mutation $(6.3 \%$ vs $3.5 \%, \mathrm{p}=.046)$ compared with CRCs with stromal POSTN-low expression. However, the frequency of MSI-high and KRAS mutation were not statistically associated with stromal POSTN expression.

\section{Prognostic implication of stromal POSTN expression in CRCs}

When we performed univariate survival analysis, CRCs with

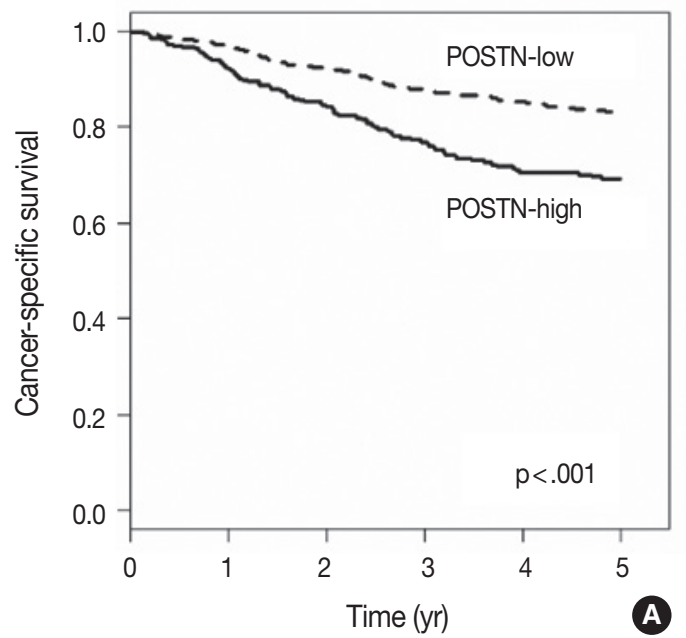

stromal POSTN-high expression showed worse 5-year PFS (HR, 1.80; 95\% CI, 1.47 to 2.20; $\mathrm{p}<.001$ ) (Fig. 2A) and worse 5-year CSS (HR, 2.00; 95\% CI, 1.52 to 2.63; p <.001) (Fig. 2B) compared with CRCs with stromal POSTN-low expression. In multivariate survival analysis, stromal POSTN-high expression was an independent prognostic indicator of poor 5-year CSS (HR, $1.50 ; 95 \% \mathrm{CI}, 1.13$ to $2.00 ; \mathrm{p}=.006$ ) (Table 2) and poor 5 -year PFS (HR, 1.38; 95\% CI, 1.12 to $1.70 ; \mathrm{p}=.003$ ) (Table 3).

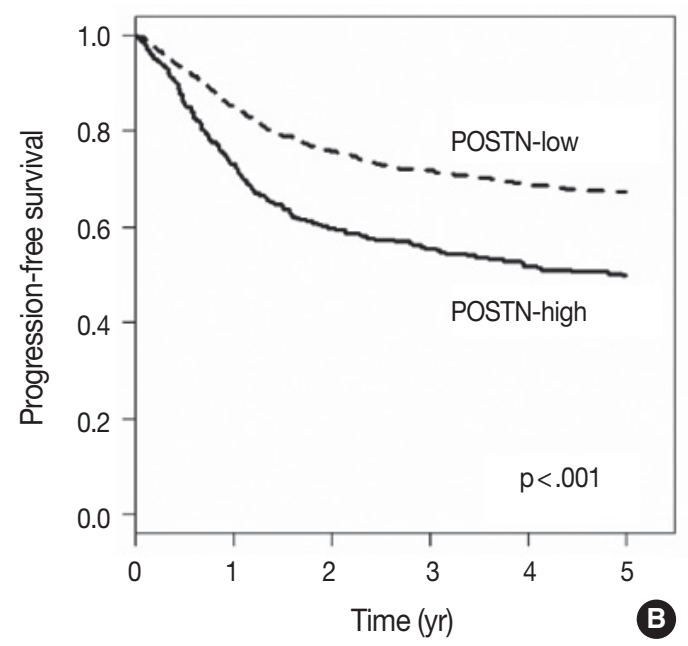

Fig. 2. Kaplan-Meier survival curves according to the stromal periostin (POSTN) expression. (A) Five-year cancer-specific survival. (B) Fiveyear progression-free survival.

Table 2. Univariate and multivariate analysis with respect to 5-year cancer-specific survival

\begin{tabular}{|c|c|c|c|c|}
\hline \multirow{2}{*}{ Variable } & \multicolumn{2}{|c|}{ Univariate } & \multicolumn{2}{|c|}{ Multivariate } \\
\hline & $\mathrm{HR}$ & $p$-value & $\mathrm{HR}$ & $p$-value \\
\hline Gross (infiltrative/fungating) & $1.87(1.32-2.66)$ & $<.001$ & $1.64(1.24-2.18)$ & .001 \\
\hline Stage (III, IV/I, II) & $4.52(3.26-6.26)$ & $<.001$ & $6.30(4.27-9.29)$ & $<.001$ \\
\hline Differentiation (PD/WD, MD) & $3.41(2.05-5.67)$ & $<.001$ & $2.15(1.27-3.62)$ & .013 \\
\hline BRAF (mutant/wild type) & $1.92(1.15-3.19)$ & .012 & $1.92(1.11-3.34)$ & .041 \\
\hline Chemotherapy (treated/not-treated) & $0.94(0.71-1.25)$ & .655 & $0.38(0.28-0.52)$ & $<.001$ \\
\hline POSTN (high/low) & $2.00(1.52-2.64)$ & $<.001$ & $1.50(1.13-2.00)$ & .006 \\
\hline Tumor location (right/left) & $1.56(1.19-2.06)$ & .002 & $1.46(1.09-1.96)$ & .011 \\
\hline Tumor-infiltrating lymphocytes (high/low) & $0.69(0.49-0.97)$ & .032 & - & .051 \\
\hline Budding (present/absent) & $1.90(1.36-2.66)$ & $<.001$ & - & .129 \\
\hline Crohn-like reaction (present/absent) & $0.71(0.48-1.06)$ & .095 & - & .305 \\
\hline Age $(\geq 65 \mathrm{yr} /<65 \mathrm{yr})$ & $1.49(1.15-1.93)$ & .003 & - & .053 \\
\hline CIMP (CIMP-H/CIMP-O, L) & $1.91(1.21-3.02)$ & .006 & - & .632 \\
\hline Sex (male/female) & $1.04(0.80-1.36)$ & .762 & - & - \\
\hline Necrosis (present/absent) & $1.11(0.70-1.78)$ & .652 & - & - \\
\hline Serration (present/absent) & $1.42(0.80-2.54)$ & .235 & - & - \\
\hline Mucin (present/absent) & $1.32(0.92-1.90)$ & .136 & - & - \\
\hline MSI (MSI-H/MSS, MSI-L) & $0.82(0.48-1.41)$ & .476 & - & - \\
\hline KRAS (mutant/wild type) & $1.12(0.84-1.49)$ & .440 & - & - \\
\hline
\end{tabular}

HR, hazard ratio; PD, poorly differentiated; WD, well differentiated; MD, moderately differentiated; POSTN, periostin; CIMP, CpG island methylator phenotype; CIMP-H, CIMP-high; CIMP-L, CIMP-low; MSI, microsatellite instability; MSI-H, MSI-high; MSS, microsatellite stable; MSI-L, MSI-low. 
Table 3. Univariate and multivariate analysis with respect to 5-year progression-free survival

\begin{tabular}{|c|c|c|c|c|}
\hline \multirow{2}{*}{ Variable } & \multicolumn{2}{|c|}{ Univariate } & \multicolumn{2}{|c|}{ Multivariate } \\
\hline & $\mathrm{HR}(95 \% \mathrm{Cl})$ & $p$-value & $\mathrm{HR}(95 \% \mathrm{Cl})$ & $p$-value \\
\hline Gross (infiltrative/fungating) & $1.96(1.61-2.39)$ & $<.001$ & $1.43(1.17-1.76)$ & .001 \\
\hline Stage (III, IV/I, II) & $4.72(3.71-6.02)$ & $<.001$ & $4.75(3.63-6.23)$ & $<.001$ \\
\hline Differentiation (PD/WD, MD) & $3.32(2.28-4.85)$ & $<.001$ & $1.85(1.26-2.71)$ & .002 \\
\hline BRAF (mutant/wild type) & $1.61(1.06-2.45)$ & .027 & $1.72(1.12-2.63)$ & .013 \\
\hline Chemotherapy (treated/not-treated) & $1.40(1.11-1.76)$ & .004 & $0.59(0.46-0.76)$ & $<.001$ \\
\hline POSTN (high/low) & $1.80(1.47-2.20)$ & $<.001$ & $1.40(1.08-1.83)$ & .012 \\
\hline Budding (present/absent) & $2.15(1.66-2.79)$ & $<.001$ & $1.51(1.15-1.97)$ & .003 \\
\hline Crohn-like reaction (present/absent) & $0.72(0.53-0.98)$ & .034 & - & .302 \\
\hline Tumor-infiltrating lymphocytes (high/low) & $0.70(0.55-0.89)$ & .004 & - & .089 \\
\hline Tumor location (right/left) & $1.22(0.98-1.51)$ & .080 & - & .773 \\
\hline Age $(\geq 6 \mathrm{yr} /<65 \mathrm{yr})$ & $1.22(1.00-1.49)$ & .046 & - & .098 \\
\hline CIMP (CIMP-H/CIMP-0, L) & $1.44(0.98-2.12)$ & .061 & - & .277 \\
\hline Sex (male/female) & $0.94(0.77-1.14)$ & .514 & - & - \\
\hline Necrosis (present/absent) & $1.14(0.80-1.62)$ & .463 & - & - \\
\hline Serration (present/absent) & $1.32(0.83-2.08)$ & .244 & - & - \\
\hline Mucin (present/absent) & $1.12(0.84-1.50)$ & .448 & - & - \\
\hline MSI (MSI-H/MSS, MSI-L) & $0.80(0.54-1.20)$ & .285 & - & - \\
\hline KRAS (mutant/wild type) & $1.05(0.85-1.31)$ & .633 & - & - \\
\hline
\end{tabular}

$\mathrm{HR}$, hazard ratio; $\mathrm{Cl}$, confidence interval; PD, poorly differentiated; WD, well differentiated; MD, moderately differentiated; POSTN, periostin; CIMP, CpG island methylator phenotype; CIMP-H, CIMP-high; MSI, microsatellite instability; MSI-H, MSI-high; MSS, microsatellite stable; MSI-L, MSI-low.

\section{DISCUSSION}

Recent studies show POSTN is overexpressed in cancer stroma of various malignancies, including colorectal, breast, lung, pancreatic, ovarian, gastric, head and neck, thyroid and prostate cancer as well as in glioblastoma. ${ }^{19}$ POSTN is thought to play important roles in tumorigenesis, such as invasiveness, metastasis, angiogenesis, lymphangiogenesis, and chemoresistance. ${ }^{8,15,20-24}$ In this study, we found that POSTN is overexpressed in 304 (27.0\%) out of 1,125 cases, which is consistent with the results of other studies in several malignancies. ${ }^{19}$ Our study demonstrated that high POSTN expression is correlated with several aggressive clinicopathological features of CRCs. First, stromal POSTN expression was higher in CRCs of infiltrative growth pattern than CRCs of fungating growth pattern, which implies that POSTN is involved in infiltrative growth of tumor. Second, POSTN expression was correlated with $\mathrm{T}, \mathrm{N}$, or $\mathrm{M}$ category. These results suggest that POSTN might participate in tumor progression and nodal or distant metastasis, which is consistent with the results of a recent study that POSTN expression is higher in distant metastatic lesion than in matched primary colorectal lesion. ${ }^{17}$ Cell line studies demonstrated that POSTN induces invasive activity and bestows a metastatic potential to HEK 293 T cells which are tumorigenic but nonmetastatic. ${ }^{11,17}$ These results support the idea that POSTN might contribute to metastasis of tumor cells. Third, high tumor budding status was correlated with high POSTN expression. Several studies addressed that tumor budding should be regarded as a biomarker of poor prognosis, correlated with epithelial mesenchymal transition. ${ }^{25-28}$ A cell line study has demonstrated that stable transfection of POSTN induced epithelial-mesenchymal transition and increased invasive activity in HEK $293 \mathrm{~T}$ cells. ${ }^{11}$ However, our present study has several limitations. First, this study is a retrospective study in a single institution. Second, stromal POSTN expression was evaluated in tissue microarray.

In our study, stromal POSTN expression was associated with CIMP-high and BRAF mutation. Because CIMP-high and BRAF mutation are hallmarks of molecular alterations in serrated neoplasia pathway, we can presume that stromal POSTN overexpression is associated with serrated neoplasia pathway. In a study by De Sousa et al., ${ }^{29}$ CCS3 subtype, which is related with serrated neoplasia pathway, showed overexpression of genes associated with epithelial mesenchymal transition and matrix remodeling. Recently, Fessler et al..$^{0}$ demonstrated the induction of a mesenchymal phenotype upon transforming growth factor $\beta$ treatment in a genetically engineered organoid culture carrying BRAF V600E mutation. In serrated neoplasia pathway, three molecular groups are known to be involved. One is BRAFmutant/CIMP-high/ MSI-high subgroup, another is BRAFmutant/CIMP-high/microsatellite stable (MSS) subgroup, and 
the other is KRAS-mutant/CIMP-low/MSS subgroup. ${ }^{31,32}$ In this study, stromal POSTN overexpression was related with $B R A F$-mutant/CIMP-high/MSS subgroup (data not shown). Moreover, some clinicopathological features associated with CIMP, such as right colon preponderance, poor differentiation, high rates of tumor budding, frequent lymphatic, vascular, or perineural invasion and nodal metastasis, were more frequently observed in POSTN-high colon cancer compared with POSTNlow colon cancer. Further molecular test is needed to elucidate the relation between high POSTN stromal expression and serrated neoplasia pathway.

An accumulating series of study results indicate that cancerassociated fibroblasts contribute to chemo-resistance and poor patient survival. ${ }^{15,17,28}$ According to Xu et al., medium to high stromal POSTN expression was an independent predictor of poor disease-free survival and disease-specific survival. They also presented the association of chemo-resistance and stromal POSTN expression in stage II CRCs and stage III CRCs with chemotherapy history. In in vitro study, SW480 and HT29 cells cultured in stromal POSTN-enriched media showed chemoresistance to 5-FU. Recombinant POSTN increased proliferation and phosphorylation of Akt in SW480 and HT29 cells. Furthermore, the proliferation and phosphorylation of Akt were inhibited by phosphoinositide 3-kinase inhibitor LY294002. ${ }^{17,24}$ In our present study, stromal POSTN-high expression was an independent prognostic indicator of poor 5-year CSS and 5-year PFS. However, we could not confirm the chemo-resistance effect of stromal POSTN expression in our cohort (data not shown).

In conclusion, our findings suggest that immunohistochemical evaluation of stromal POSTN expression could be utilized for the identification of a subset of CRCs with poor prognosis. To determine whether stromal POSTN expression status is a prognostic marker of CRC, further studies are needed to validate this finding with an independent series of large-scaled CRC cases.

\section{Conflicts of Interest}

No potential conflict of interest relevant to this article was reported.

\section{REFERENCES}

1. Ferlay J, Soerjomataram I, Dikshit R, et al. Cancer incidence and mortality worldwide: sources, methods and major patterns in GLOBOCAN 2012. Int J Cancer 2015; 136: E359-86.

2. O'Connell MJ, Campbell ME, Goldberg RM, et al. Survival follow- ing recurrence in stage II and III colon cancer: findings from the ACCENT data set. J Clin Oncol 2008; 26: 2336-41.

3. Bozóky B, Savchenko A, Csermely P, et al. Novel signatures of cancer-associated fibroblasts. Int J Cancer 2013; 133: 286-93.

4. Chen WJ, Ho CC, Chang YL, et al. Cancer-associated fibroblasts regulate the plasticity of lung cancer stemness via paracrine signalling. Nat Commun 2014; 5: 3472.

5. Huang L, Xu AM, Liu S, Liu W, Li TJ. Cancer-associated fibroblasts in digestive tumors. World J Gastroenterol 2014; 20: 17804-18.

6. Hwang RF, Moore T, Arumugam T, et al. Cancer-associated stromal fibroblasts promote pancreatic tumor progression. Cancer Res 2008; 68: 918-26.

7. Pankova D, Chen Y, Terajima M, et al. Cancer-associated fibroblasts induce a collagen cross-link switch in tumor stroma. Mol Cancer Res 2016; 14: 287-95.

8. Morra L, Moch H. Periostin expression and epithelial-mesenchymal transition in cancer: a review and an update. Virchows Arch 2011; 459: 465-75.

9. Kikuchi Y, Kashima TG, Nishiyama T, et al. Periostin is expressed in pericryptal fibroblasts and cancer-associated fibroblasts in the colon. J Histochem Cytochem 2008; 56: 753-64.

10. Fukushima N, Kikuchi Y, Nishiyama T, Kudo A, Fukayama M. Periostin deposition in the stroma of invasive and intraductal neoplasms of the pancreas. Mod Pathol 2008; 21: 1044-53.

11. Yan W, Shao R. Transduction of a mesenchyme-specific gene periostin into $293 \mathrm{~T}$ cells induces cell invasive activity through epithelial-mesenchymal transformation. J Biol Chem 2006; 281: 19700-8.

12. Gillan L, Matei D, Fishman DA, Gerbin CS, Karlan BY, Chang DD. Periostin secreted by epithelial ovarian carcinoma is a ligand for $\alpha \mathrm{V} \beta 3$ and $\alpha \mathrm{V} \beta 5$ integrins and promotes cell motility. Cancer Res 2002; 62: 5358-64.

13. Baril P, Gangeswaran R, Mahon PC, et al. Periostin promotes invasiveness and resistance of pancreatic cancer cells to hypoxia-induced cell death: role of the beta4 integrin and the PI3k pathway. Oncogene 2007; 26: 2082-94.

14. Bao S, Ouyang G, Bai X, et al. Periostin potently promotes metastatic growth of colon cancer by augmenting cell survival via the Akt/PKB pathway. Cancer Cell 2004; 5: 329-39.

15. Sung PL, Jan YH, Lin SC, et al. Periostin in tumor microenvironment is associated with poor prognosis and platinum resistance in epithelial ovarian carcinoma. Oncotarget 2016; 7: 4036-47.

16. Underwood TJ, Hayden AL, Derouet M, et al. Cancer-associated fibroblasts predict poor outcome and promote periostin-dependent invasion in oesophageal adenocarcinoma. J Pathol 2015; 235: 466-77.

17. Xu X, Chang W, Yuan J, et al. Periostin expression in intra-tumoral stromal cells is prognostic and predictive for colorectal carcinoma 
via creating a cancer-supportive niche. Oncotarget 2016; 7: 798-813.

18. Kim JH, Shin SH, Kwon HJ, Cho NY, Kang GH. Prognostic implications of $\mathrm{CpG}$ island hypermethylator phenotype in colorectal cancers. Virchows Arch 2009; 455: 485-94.

19. Ratajczak-Wielgomas K, Dziegiel P. The role of periostin in neoplastic processes. Folia Histochem Cytobiol 2015; 53: 120-32.

20. Kyutoku M, Taniyama Y, Katsuragi N, et al. Role of periostin in cancer progression and metastasis: inhibition of breast cancer progression and metastasis by anti-periostin antibody in a murine model. Int J Mol Med 2011; 28: 181-6.

21. Shao R, Bao S, Bai X, et al. Acquired expression of periostin by human breast cancers promotes tumor angiogenesis through up-regulation of vascular endothelial growth factor receptor 2 expression. Mol Cell Biol 2004; 24: 3992-4003.

22. Takanami I, Abiko T, Koizumi S. Expression of periostin in patients with non-small cell lung cancer: correlation with angiogenesis and lymphangiogenesis. Int J Biol Markers 2008; 23: 182-6.

23. Wu G, Wang X, Zhang X. Clinical implications of periostin in the liver metastasis of colorectal cancer. Cancer Biother Radiopharm 2013; 28: 298-302.

24. Xiao ZM, Wang XY, Wang AM. Periostin induces chemoresistance in colon cancer cells through activation of the PI3K/Akt/survivin pathway. Biotechnol Appl Biochem 2015; 62: 401-6.

25. Dawson H, Lugli A. Molecular and pathogenetic aspects of tumor budding in colorectal cancer. Front Med (Lausanne) 2015; 2: 11.

26. Zheng X, Carstens JL, Kim J, et al. Epithelial-to-mesenchymal transition is dispensable for metastasis but induces chemoresistance in pancreatic cancer. Nature 2015; 527: 525-30.

27. Koelzer VH, Dawson H, Andersson E, et al. Active immunosurveillance in the tumor microenvironment of colorectal cancer is associated with low frequency tumor budding and improved outcome. Transl Res 2015; 166: 207-17.

28. Ueno $\mathrm{H}$, Shinto E, Shimazaki $\mathrm{H}$, et al. Histologic categorization of desmoplastic reaction: its relevance to the colorectal cancer microenvironment and prognosis. Ann Surg Oncol 2015; 22: 1504-12.

29. De Sousa EM, Wang X, Jansen M, et al. Poor-prognosis colon cancer is defined by a molecularly distinct subtype and develops from serrated precursor lesions. Nat Med 2013; 19: 614-8.

30. Fessler E, Drost J, van Hooff SR, et al. TGFbeta signaling directs serrated adenomas to the mesenchymal colorectal cancer subtype. EMBO Mol Med 2016; 8: 745-60.

31. Kim JH, Bae JM, Cho NY, Kang GH. Distinct features between MLH1-methylated and unmethylated colorectal carcinomas with the $\mathrm{CpG}$ island methylator phenotype: implications in the serrated neoplasia pathway. Oncotarget 2016; 7: 14095-111.

32. Tsai JH, Cheng $\mathrm{CH}$, Chen CC, et al. Traditional serrated adenoma with $B R A F$ mutation is associated with synchronous/metachronous BRAF-mutated serrated lesions. Histopathology 2016; 68: 810-8. 